\title{
CORRECTION
}

\section{Correction to: Selective kappa-opioid antagonism ameliorates anhedonic behavior: evidence from the Fast-fail Trial in Mood and Anxiety Spectrum Disorders (FAST-MAS)}

Diego A. Pizzagalli (D), Moria Smoski, Yuen-Siang Ang, Alexis E. Whitton (D), Gerard Sanacora, Sanjay J. Mathew (D), John Nurnberger Jr, Sarah H. Lisanby, Dan V. losifescu, James W. Murrough, Hongqiu Yang, Richard D. Weiner, Joseph R. Calabrese iD, Wayne Goodman, William Z. Potter and Andrew D. Krystal (iD

() The Author(s), under exclusive licence to American College of Neuropsychopharmacology 2021

Neuropsychopharmacology (2021) 46:2224; https://doi.org/10.1038/s41386-021-01145-9

Correction to: Neuropsychopharmacology https://doi.org/10.1038/ s41386-020-0738-4, published online 16 June 2020

In this article a conflict of interest was missing.
The co-author Sanjay J. Mathew served as a consultant to Alkermes.

The original article has been corrected. 